EPJ Web of Conferences 16, 06005 (2011)

DOI: $10.1051 /$ epjconf/20111606005

(C) Owned by the authors, published by EDP Sciences, 2011

\title{
Extending the Canada-France Brown Dwarf Survey to the near infrared
}

\author{
P. Delorme ${ }^{1,4, a}$, L. Albert ${ }^{2}$, E. Artigau ${ }^{3,9}$, T. Forveille ${ }^{4}$, X. Delfosse ${ }^{4}$, C. Reylé ${ }^{5}$, \\ C.J. Willott ${ }^{6}$, E. Bertin ${ }^{7}$ and F. Allard ${ }^{8}$ \\ 1 School of Physics \& Astronomy, University of St Andrews North Haugh, St Andrews \\ KY16 9SS, Scotland \\ 2 Canada-France-Hawaii Telescope Corporation, 65-1238 Mamalahoa Highway, Kamuela, \\ HI96743, USA \\ 3 Département de physique and Observatoire du Mont Mégantic, Université de Montréal, \\ CP. 6128, Succursale Centre-Ville, Montréal, QC H3C 3J7, Canada \\ 4 Laboratoire d'Astrophysique de Grenoble, UMR5571 (CNRS and Université J. Fourier), \\ BP. 53, 38041 Grenoble Cedex 9, France \\ 5 Observatoire de Besançon, Institut Utinam, UMR CNRS 6213, BP. 1615, 25010 Besançon \\ Cedex, France \\ 6 University of Ottawa, Physics Department, 150 Louis Pasteur, MacDonald Hall, Ottawa, \\ ON K1N 6N5, Canada \\ 7 Institut d'Astrophysique de Paris-CNRS, 98bis Boulevard Arago, 75014 Paris, France \\ 8 C.R.A.L. (UMR 5574 CNRS), École Normale Supérieure, 69364 Lyon Cedex 07, France \\ 9 Gemini Observatory Southern Operations Center clo AURA, Casilla 603 La Serena, Chile
}

\begin{abstract}
We present the first results of the Canada-France Brown Dwarfs Survey-InfraRed, hereafter CFBDSIR, our near infrared extension to the optical wide field survey CFBDS. Our final objectives are to constrain ultracool atmosphere physics by finding a statistically significant sample of objects cooler than $600 \mathrm{~K}$ and to explore the ultracool brown dwarf mass function building on a well defined sample of such objects. We identify candidates in CFHT/Wircam $J$ and CFHT/MegaCam $z^{\prime}$ images using optimized psf-fitting within Source Extractor, and follow them up with pointed near infrared imaging with SOFI at NTT. We have so far analysed and followed up all candidates on the first 66 square degrees of the 280 square degrees survey. We identified $64 \mathrm{~T}$ dwarfs candidates with $z^{\prime}-J>3.5$ and have confirmed 3 of them as ultracool brown dwarfs (later than T7 dwarfs and Y dwarfs candidates), and 14 of them as early and mid-T dwarfs based on their far red and NIR colours. We also present here the NIR spectra of one of these ultracool dwarfs, CFBDSIR1458 which confirms it as one of the coldest brown dwarf known, in the $500-600 \mathrm{~K}$ temperature range.
\end{abstract}

\section{INTRODUCTION}

The significant improvment in detector technology and data storage and analysis facilities in the past decade made it possible to carry out wide field surveys covering a large fraction of the sky instead of targeting specific sources. The wealth of data from these surveys necessitate a complex dedicated computer analysis to single out relevant scientific information. These surveys, such as DENIS [Epchtein et al., 1997], SDSS [York et al., 2000], 2MASS [Skrutskie et al., 2006], UKIDSS [Lawrence et al., 2007]

ae-mail: pd10@st-andrews.ac.uk

This is an Open Access article distributed under the terms of the Creative Commons Attribution-Noncommercial License 3.0, which permits unrestricted use, distribution, and reproduction in any noncommercial medium, provided the original work is properly cited. 


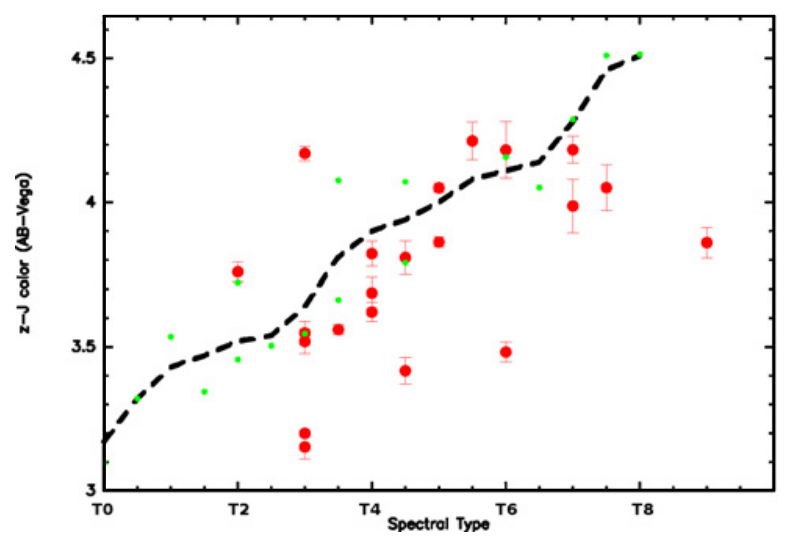

Figure 1. $z^{\prime}-J$ color spectral type relation. In green are the synthetic Megacam/Wircam colors derived from known objects with publicly available spectra from http://www.jach.hawaii.edu/ skl/LTdata.html, The black dashed line is the resulting averaged color-spectral type relation. Red points show the observational colors and spectral types of CFBDS spectroscopically confirmed T dwarfs.

and CFBDS [Delorme et al., 2008b] contain hundreds of millions of astrophysical sources and lead to many advances in various fields, notably to identify extremely rare objects and build robust statistical studies.

The Canada-France Brown Dwarf Survey is a far-red wide-field survey using Megacam at CFHT that discovered more than $70 \mathrm{~T}$ dwarfs and $300 \mathrm{~L}$ dwarfs, including some of the coldest known brown dwarfs. The candidates were identified thanks to their very red $i^{\prime}-z^{\prime}$ colour. In order to increase its sensitivity to the latest type brown dwarfs whose emission peaks in the infrared, we conducted a shallow Wircam survey in the $J$-band on the field observed by CFBDS. This survey is targeted at finding ultracool brown dwarfs $\left(\mathrm{T}_{e f f}<650 \mathrm{~K}\right.$ ) of which only 5 are currently published, by Burningham et al., [2008, 2009], Delorme et al., [2008a], Warren et al., [2007]. These rare objects are in many ways the intermediate "missing link" between the cold atmospheres of the Solar System's giant planets (at around $100 \mathrm{~K}$ ) and cold stellar atmospheres. The physics and chemistry of their cool atmospheres, dominated by broad molecular absorption bands, are very planetary like Kulkarni [1997, for instance] and the spectra obtained on cool brown dwarfs are key to constrain the atmosphere models that aim at modelizing planetary and stellar atmospheres.

We present here the observations and data analysis strategies at the core of the survey (section 2), before focusing in section 3 on the first ultracool brown dwarfs identified with CFBDSIR.

\section{THE CFBD SURVEY-INFRARED}

\subsection{Survey rational}

The CFBDSIR, identifies astrophysical sources on $J$-band Wircam [Puget et al., 2004] images and selects ultracool brown dwarfs candidates depending upon their $z^{\prime}-J$ colors, using $z^{\prime}$ magnitudes from CFBDS MegaCam [Boulade et al., 2003] images. The $z^{\prime}-J$ colour has excellent dynamics for brown dwarfs, varying from 2.6 to over 4.5 from mid-L and late-T (Fig. 1). It therefore provides (at least at high $\mathrm{S} / \mathrm{N}$ ratio) a good selection criteria to identify ultracool brown dwarfs. They usually have $z^{\prime}-J>3.8$ as confirmed by atmosphere models such as BT-Settl [Allard et al. 2007], synthetic Megacam/Wircam colors derived from known objects [Delorme et al., 2008b] and direct observational data [Burningham et al., 2008, Delorme et al., 2008a],. Models expect cooler objects, not yet discovered $(\mathrm{T}<550 \mathrm{~K})$, to be even redder. It is however visible in figure 1 that the spread in the color-spectral type relation for late $\mathrm{T}$ dwarfs is high, meaning that this $z^{\prime}-J$ color range is also populated by warmer mid T dwarfs. 
Follow-up photometry of candidates in $H$ and $K s$ band allows to discriminate relatively blue very late $\mathrm{T}$ from the red mid-T dwarfs [See Lodieu et al., 2007, for instance]. Only very rare astrophysical contaminants can pollute this color range: QSOs with $z>7$ of which none have been discovered yet and atypicals broad absorption lines quasars at relatively high redshift $(z \sim>2)$, such as the one described in Hall et al., [2002]. However these objects can be discriminated from brown dwarfs with photometry only : they are not as red in far red than ultracool brown dwarfs and will appear in the $i^{\prime}$ CFBDS images whereas brown dwarfs will not.

\subsection{Observations}

We obtained Wircam imaging of $\sim 280$ square degrees with an average depth of $J_{v e g a}=20.0$ for a $10 \sigma$ point source detection, ensuring that photometry is accurate and spurious sources problems are low. The data has been acquired at CFHT atop Mauna Kea, with a seeing varying from 0.6" to 1.0" during semester 2006B, 2007A and 2009B. These Wircam pointings targeted 280 existing Megacam fields from CFBDS, that have already been observed in $i^{\prime}$ and $z^{\prime}$. Each $\sim 1$ square degree Megacam field was covered with nine $21^{\prime}$ by $21^{\prime}$ Wircam $J$-band $2 \times 45$ seconds exposures that were stacked together to produce 1 square degree Wircam frames matching the original Megacam field. The whole 280 square degrees area was then covered in 80 hours of CFHT telescope time, resulting in 90 seconds stacked exposures of the whole area. The relative shallowness of these 90 seconds exposure time ensures that all confirmed ultracool brown dwarf candidates can be observed with low resolution spectrograph on 8 meters-class telescopes.

\subsection{Analysis and follow up}

Since CFBDSIR $J$-band images contain more than 5000000 sources among which we hope to find about 10 ultracool brown dwarf we need to carry out a very robust analysis: a false detection rates of even 1 per $10^{4}$ real sources would flood true brown dwarfs in our candidate lists. We use the point spread function analysis described in Delorme et al., [2008b] to analysis both $J$ and $z^{\prime}$ images and then crossmatch the two catalogs to derive the $z^{\prime}-J$ colors of all sources in the images. We select objects with a signal to noise better than 10 and $z^{\prime}-J>3.5$, a very conservative color limit to be sure to select all ultracool brown dwarfs whose expected $z^{\prime}-J$ color is usually over 3.8. Since brown dwarfs are point sources and that most instrumental artefacts (mainly bad pixels and cross-talk remaining features) are not, we use the $\chi^{2}$ from the psf-fitting to eliminate most of the artefacts from our candidate list.

All the selected candidates, numbering about one per square degree are then followed-up by pointed NIR observations with SOFI camera at NTT. This allows to weed out any remaining artefacts and to better constrain the spectral type of the brown dwarfs we selected. We retain the blue candidates, with $J-H<0.1$ as our ultracool brown dwarfs candidates to further characterize with Near InfraRed low resolution spectroscopic observations with $8 \mathrm{~m}$ class telescopes.

\section{RESULTS}

\subsection{Photometry}

We analysed $z^{\prime}$ and $J$ images on most of the 280 square degrees of the survey but only the candidates found on a 66 square degree subset of the data have been completely followed-up in photometry. We obtained pointed NIR observations of all 64 candidates selected on these subset and confirmed $14 \mathrm{~T}$ dwarfs, of which 3 are robust ultracool brown dwarf candidates. Those are either very late $\mathrm{T}$ dwarfs (>T8), or cooler Y dwarfs.

Extrapolating these results to 280 square degree, we expect to find $\sim 60 \mathrm{mid} / \mathrm{late} \mathrm{T}$ dwarfs and $\sim 10$ ultracool brown dwarfs with CFBDSIR. Our ultracool brown dwarfs candidates need spectroscopic NIR 
EPJ Web of Conferences

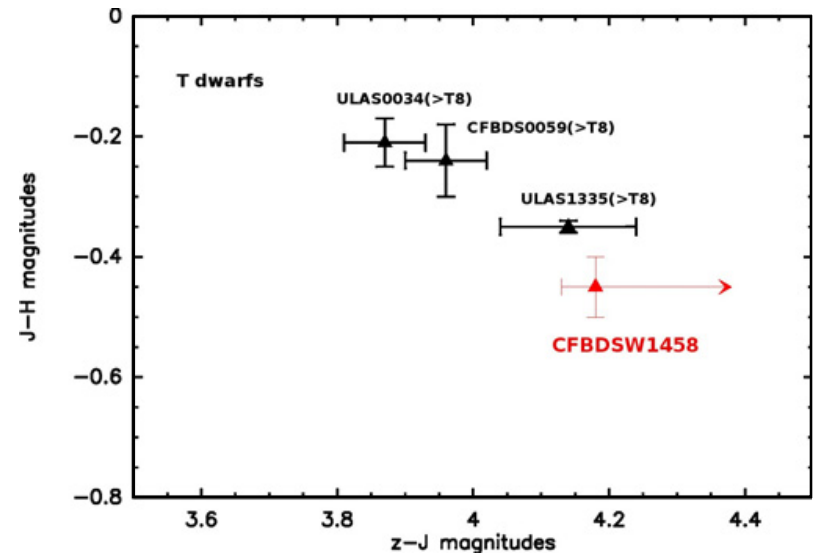

Figure 2. Colour-colour diagram of the latest brown dwarfs known, $>\mathrm{T} 8$, later than spectrum used for up to date Spectral Classification Scheme. The regular T dwarf colour range is highlighted on the upper left corner and CFBDSIR1458 is highlighted on the lower right corner.

\begin{tabular}{|c|c|c|c|c|}
\hline Object & Sp. Type & $\mathrm{H}_{2} \mathrm{O}-\mathrm{H}$ & $\mathrm{CH}_{4}-\mathrm{H}$ & $\mathrm{NH}_{3}-\mathrm{H}$ \\
\hline 2mass0415 & T8 & 0.183 & 0.104 & 0.625 \\
\hline ULAS0034 & T8+/Y? & 0.133 & 0.096 & 0.516 \\
\hline CFBDS0059 & T8+/Y? & 0.119 & 0.084 & 0.526 \\
\hline ULAS1335 & T8+/Y? & 0.114 & 0.077 & 0.564 \\
\hline CFBDS-W1458 & T8+/Y? & 0.122 & 0.011 & 0.516 \\
\hline
\end{tabular}

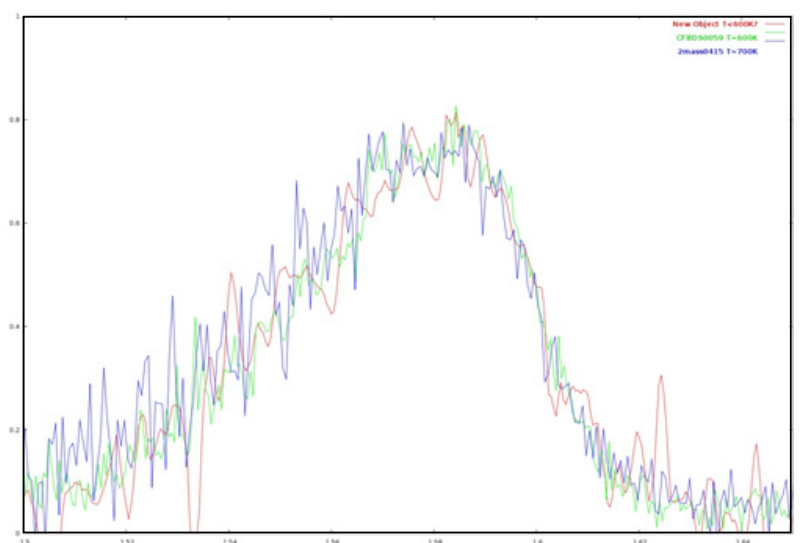

Figure 3. CFBDS-W1458 spectrum (red, T8+/Y?), together with the spectra of CFBDS0059 (green, T8+/Y?, probable ammonia absorption) and 2 mass 0415 (blue, T8). Several features that differenciate 2 mass0415 from the cooler CFBDS0059 are even more marked on our target, notably at $1.55,1.56$ and $1.57 \mu \mathrm{m}$. This advocates that CFBDSIR1458 could be even colder than CFBDS0059.

low-resolution observations to derive an accurate spectral type and characterize their cool atmosphere physics.

\subsection{Spectroscopic confirmation of one new ultracool brown dwarf}

Up to know only one of our photometrically confirmed (See Fig. 2) ultracool brown dwarfs candidates have been observed in spectroscopy. The observational properties of this extreme object, 
CFBDSIR-145829+101343, hereafter CFBDSIR-145829 + 101343, are summarized here:

- Very red far-red colours: $z^{\prime}-J>4.18(5 \sigma)$. The object is so red that it is undetected to $z=23.8$ (as well as at $i^{\prime}$ and $r^{\prime}$ ), and its $z^{\prime}-J$ color is thus a lower limit. For comparison, Leggett et al (2009), find $z^{\prime}-J=3.96,3.87$ and 4.14 for ULAS J0034 - 00, CFBD J0059 - 01 and ULAS $\mathrm{J} 1335+11$. The object is thus robustly redder at $z^{\prime}-J$ than any previously known brown dwarf.

- Very blue NIR colours, with $[J-H ; J-K s]=[-0.45 ;-0.80]$, pointing to very strong molecular absorptions in the $H$ and $K$ bands. For comparison, ULAS J0034 - 00, CFBD J005901 and ULAS J1335 +11 respectively have $[J-H ; J-K S]=[-0.24 ;-0.23],[-0.21 ;-0.57]$ and $[-0.35 ;-0.38]$ (Leggett et al, 2009).

- Extreme spectroscopic indices derived from an ISAAC $H$-band spectra obtained with DDT observations at ESO VLT, see table 1.

The resulting spectrum (Fig. 3) has a low signal to noise due to the fainteness of the target ( $J_{v e g a}=19.6$ ) and its relatively high airmass at the time of the observations. We plan to obtain better signal to noise observations but with the present spectrum were already able to derive the spectroscopic indices described by Burgasser et al., [2006], Delorme et al., [2008a], Warren et al., [2007] which quantify the strength of key molecular absorption bands. This classifies CFBDS1458 as an ultracool brown dwarfs with spectral type later than T8 and a temperature in the same range $(\sim 500-600 \mathrm{~K})$ as the coolest brown dwarfs known.

\section{References}

Allard, F., Allard, N. F., Homeier, D., et al. 2007, A\&A, 474, L21

Boulade, O., Charlot, X., Abbon, P., et al. 2003, in Presented at the Society of Photo-Optical Instrumentation Engineers (SPIE) Conference, Vol. 4841, Instrument Design and Performance for Optical/Infrared Ground-based Telescopes. Edited by Iye, Masanori; Moorwood, Alan F. M. Proceedings of the SPIE, Volume 4841, pp. 72-81 (2003)., ed. M. Iye \& A. F. M. Moorwood, 72-81

Burgasser, A. J., Geballe, T. R., Leggett, S. K., Kirkpatrick, J. D., \& Golimowski, D. A. 2006, ApJ, 637, 1067

Burningham, B., Pinfield, D. J., Leggett, S. K., et al. 2008, MNRAS, 391, 320

Burningham, B., Pinfield, D. J., Leggett, S. K., et al. 2009, MNRAS, 395, 1237

Delorme, P., Delfosse, X., Albert, L., \& al. 2008a, A\&A, 482, 961

Delorme, P., Willott, C. J., Forveille, T., et al. 2008b, A\&A, 484, 469

Epchtein, N., de Batz, B., Capoani, L., et al. 1997, The Messenger, 87, 27

Hall, P. B., Anderson, S. F., Strauss, M. A., et al. 2002, ApJs, 141, 267

Kulkarni, S. R. 1997, Science, 276, 1350

Lawrence, A., Warren, S. J., Almaini, O., et al. 2007, MNRAS, 379, 1599

Lodieu, N., Pinfield, D. J., Leggett, S. K., et al. 2007, MNRAS, 379, 1423

Puget, P., Stadler, E., Doyon, R., et al. 2004, in Society of Photo-Optical Instrumentation Engineers (SPIE) Conference Series, Vol. 5492, Society of Photo-Optical Instrumentation Engineers (SPIE) Conference Series, ed. A. F. M. Moorwood \& M. Iye, 978-987

Skrutskie, M. F., Cutri, R. M., Stiening, R., et al. 2006, AJ, 131, 1163

Warren, S. J., Mortlock, D. J., Leggett, S. K., \& al. 2007, MNRAS, 381, 1400

York, D. G., Adelman, J., Anderson, Jr., J. E., et al. 2000, AJ, 120, 1579 